\title{
Editorial
}

\section{Cellular senescence, aging, and age-related disease: Special issue of BMB Reports in 2019}

Cellular senescence is a state of permanent cell cycle arrest which exhibits large and flattened morphological characteristics. Cellular senescence might evolve to a beneficial process to suppress the accumulation of severely damaged cells. However, senescent cells are considered as the cause of age-related pathologies and diseases. In contrast to replicative senescence, premature senescence could be rapidly induced through intrinsic and extrinsic insults. Cancer cell senescence has been accepted as an alternative and attractive tumor suppressive mechanism. In addition to the non-proliferative aspect, senescence associated with diverse functionality affects tissue homeostasis through the communication with their neighboring cells. Aging is a time-dependent process of deterioration, accompanied by progressive metabolic alteration and accumulation of senescent cells. Thus, aging is the main risk factor for many chronic diseases. Chronic inflammation which is principally induced by senescence associated secretory phenotype (SASP) might be the major underlying cause of many age-related disease. Recently, senotherapeutics, medicines targeting to kill or modulate senescent cells selectively, have been implicated to be novel strategy for aging intervention which is applicable to promote healthy aging and to prevent or treat age-related diseases. This special issue invites one perspective and ten mini-reviews covering various senescence features, gene expression characteristics with aging, therapeutic strategies related with cancer and age-related diseases.

Dr. Masashi Narita from the University of Cambridge is invited to speak about the perspectives in this issue. The best example of non-autonomous activities of senescence is SASP. In this perspective, he mentions other form of non-autonomous activities of senescence, i.e., NOTCH-mediated direct cell-cell contact which modulates senescence development and functionality.

The metabolic features of cellular senescence may be linked to the metabolic syndromes in aging. Dr. Gyesoon Yoon and colleagues from Ajou University provide key metabolic features and regulators of cellular senescence related with mitochondrial dysfunction and anabolic deregulation. They focus on mitochondrial retrograde signaling and key players which are closely linked to senescence.

Mitochondrial free radical theory of aging (MFRTA) has been

https://doi.org/10.5483/BMBRep.2019.52.1.002

Received 14 December 2018 a common term in the aging field. However, increasing inconsistent evidences lead to criticism for MFRTA. Dr. Changhan David Lee and colleagues from the University of Southern California review previous and recent concepts regarding the roles of mitochondria in aging. They discuss the cell autonomous and non-cell autonomous mitochondrial communications which influence cellular function, homeostasis, and aging.

Sirtuin is believed to have beneficial effects on aging through the regulation of diverse cellular processes. Dr. Kyung-Jin Min and colleagues from Inha University summarize the evidences and controversies regarding the roles of Sirtuin on cellular senescence and longevity. They discuss Sirtuin requirement in the lifespan extension by calorie restriction and introduce Sirtuin activators for therapeutics in age-related diseases.

Cellular senescence is considered as a powerful suppressive mechanism of tumorigenesis. I and Dr. Seongju Lee from Inha University describe key features and molecular pathways of cellular senescence to provide insights to develop strategies for therapeutic benefit. We also review the therapeutic strategies for the induction of cancer cell senescence as a promising way of cancer therapy.

Drs. Young Hwa Kim and Tae Jun Park from Ajou University discuss the role of senescent cells in the tissues under the pathologic condition and the senescent cells-targeted therapy for cancer treatment. They also describe the technical limitations for the identification of senescent cells in vivo and pathological role of senescent cells in tumor microenvironment.

Recent studies have reported that genetic ablation of senescent cells lengthens healthy life span and reduces the risk of age-related pathologies in mouse model. Drs. Eok-Cheon Kim and Jae-Ryong Kim from Yeungnam University review the development of senotherapeutics in relation to aging and age-related disease. They introduce useful candidates for senolytics and senomorphics and discuss future directions and opportunities of senotherapeutics.

The age-related functional change is accompanied by a low-grade, unresolved chronic inflammation. Dr. Hae Young Chung and colleagues from Pusan National University, Korea Institute of Oriental Medicine, and The University of Texas Health Science Center at San Antonio describe a new integrative concept on age-related chronic inflammation to encounter broad characteristics of senescence and to better understand interactions between immune and non-immune

ISSN: 1976-670X (electronic edition)

Copyright (C) 2019 by the The Korean Society for Biochemistry and Molecular Biology

(ㄷ) This is an open-access article distributed under the terms of the Creative Commons Attribution Non-Commercial License (http://creativecommons.org/licenses/by-nc/4.0) which permits unrestricted non-commercial use, distribution, and reproduction in any medium, provided the original work is properly cited. 
cells, and metabolic and non-metabolic organs in aging. They overview senoinflammation and emphasized on modulatory effect of calorie restriction on senoinflammation to promote healthy longevity.

Sarcopenia, a loss of skeletal muscle is an inevitable process in aging. Dr. Ki-Sun Kwon and colleagues from Korea Research Institute of Bioscience and Biotechnology and University of Science and Technology describe the critical roles of AMPK-ULK1 and FoxO/PGC- $1 \alpha$ signaling pathways in autophagy induction for development of therapeutic targets in skeletal muscle aging. They also introduce the current understanding of physiological importance of autophagy activation in muscle regeneration.

Drs. Seung-Soo Kim and Cheol-Koo Lee from Korea University review physiological characteristics and global gene expression analyses in genetically modified mouse models such as growth hormone-deficient mice, insulin receptor substrate-deficient mice, and mTORC1- and MYC-reduced mice, to understand longevity mechanism.

Gene expression is tightly controlled for the homeostasis and is differentially regulated with aging. Dr. Bérénice A. Benayoun and colleagues from the University of Southern California discuss the current knowledge on the transcriptional alterations in aged metazoans. They provide informations regarding the aging transcriptome such as splicing, long noncoding RNAs, circular RNAs, small noncoding RNAs, and others to identify important regulatory targets for the healthy lifespan in humans.

It is believed that better understanding of the molecular features and the key players of cellular senescence and aging based on its physiology and pathology will provide promising therapeutic strategies for heathy life span in the future. I hope this special issue will give a help to have an interest in this field and to achieve appropriate therapeutic goals.

Jae-Seon Lee ${ }^{1,2}$, Ph.D.

${ }^{1}$ Hypoxia-related Disease Research Center, ${ }^{2}$ Department of Molecular Biology, College of Medicine, Inha University, Incheon 22212, Korea

E-mail: jaeslee@inha.ac.kr 